\title{
Interpolation of Single Beam Echo Sounder Data for 3D Bathymetric Model
}

\author{
Claudio Parente ${ }^{1}$, Andrea Vallario ${ }^{2}$ \\ Department of Sciences and Technologies \\ University of Naples "Parthenope" \\ Naples, Italy
}

\begin{abstract}
Transmitting sound waves into water, and measuring time interval between emission and return of a pulse, single beam echo sounder determines the depth of the sea. To obtain a bathymetric model representing sea-floor continuously, interpolation is necessary to process irregular spaced measured points resulting from echo sounder acquisition and calculate the depths in unsampled areas. Several interpolation methods are available in literature and the choice of the most suitable of them cannot be made a priori, but requires to be evaluated each time. This paper aims to compare different interpolation methods to process single beam echo sounder data of the Gulf of Pozzuoli (Italy) for 3D model achievement. The experiments are carried out in GIS (Geographic Information System) environment (Software: ArcGIS 10.3 and its extension Geostatistical Analyst by ESRI). The choice of the most accurate digital depth model is made using automatic cross validation. Radial basis function and kriging prove to be the best interpolation methods for the considered dataset.
\end{abstract}

Keywords-Interpolation; bathymetric model; 3D model; digital depth model; kriging; radial basis function; Geographic Information System (GIS)

\section{INTRODUCTION}

As reported in literature, interpolation is a process of using a discrete set of known data points to construct new data points $[1,2,3,4]$. Data points resulting from experimentations and measurements represent the values of a function for a limited number of values of the independent variable. Interpolation permits to estimate the values of that function for intermediate values of the independent variable $[5,6]$.

The concept of spatial interpolation is related to the digital terrain model (DTM): introduced by Miller \& Laflamme [7] at the Photogrammetry Laboratory of the Massachusetts Technology Institute, DTM can be defined as a three dimensional representation of a terrain surface consisting of $X$, $\mathrm{Y}, \mathrm{Z}$ coordinates stored in digital form. This representation can be obtained as a vector-based triangulated irregular network (TIN) as well as a grid, both displayable in 3D.

DTM represents the bare ground surface without any objects belonging to the built (power lines, buildings and towers) and natural (trees and other types of vegetation) [8]. When the earth's surface is represented including all objects on it, the model is called digital surface model (DSM). Digital elevation model (DEM) is often used as a generic term for DSM and DTM [9]. In this study, we use DEM as synonymous of DTM.
Data for DEM can be acquired using different techniques, i.e. photogrammetry, land surveying, lidar, etc. [10].

DEMs are basic in land analysis and management as they are directly usable in GIS environment [11]. They are fundamental for many applications, i.e. 3D thematic model construction [12], assessment of potential groundwater vulnerability to pollution [13], assessment of potential dam sites [14], landslide hazard [15,16], satellite images orthorectification for useful applications in coastal area studies [17], etc.

Interpolation methods that support DEM generation can be used for seabed model. This can be indicated as Digital Depth Model (DDM) because describes the variability of the distance between the sea surface and sea bottom. In other terms, interpolation methods permit to realize a bathymetric model that can be defined, according to International Hydrographic Organization (IHO), as "a digital representation of the topography (bathymetry) of the seafloor by coordinates and depths" [18]. Depth data to be processed can be obtained with different techniques, i.e. bathymetric survey, nautical map, lidar. Similarly to DEM, DDM can be generated using different interpolation methods and the choice of the one able to supply the most performed model is not banal.

For this purpose, this study aims to realize a review of some existing interpolation methods usable for DDM construction. The paper supplies suitable information to select the method for DDM generation. Firstly, the single beam echo sounder technique to acquire depth data necessary to shape seafloor is introduced. Following this, a brief description is given for some existing DEM generation methods that can be used also for DDM production. Next, the performance of each method is evaluated considering a case study concerning the Gulf of Pozzuoli (Italy): single beam echo sounder data are processed and the accuracy of each method is referred to the closeness of interpolated values to measured values. To compare the different approaches adopted in this study, cross validation is carried out for each model. Finally, we remark the importance of the work and suggest the potential applications and extensions for future studies.

\section{SOURCE OF DATA}

\section{A. Single Beam Echo Sounder}

Single beam echo sounder permits to determine the depth of water by diffusing sound waves into water. The amount of time it takes for the sound to travel through the water, spring 
back the seafloor, and return to the sounder, permits to determine the depth of water. Fundamental for this purpose is the exact knowledge of the speed of sound in water that is variable and dependent on pressure (depth), density, temperature and salinity [19,20,21]. For example, the speed of sound in water ranges from 1450 to 1498 meters per second in distilled water and $1531 \mathrm{~m} / \mathrm{s}$ in sea water at $20-25^{\circ} \mathrm{C}$ [22].

International Hydrographic Organization (IHO) has fixed the requirements for bathymetric survey, so the necessary precision and accuracy of the hydrographic echo sounder are defined in IHO special publication S-44 [18].

Hydrographic echo sounders are usually dual frequency: a low frequency pulse (normally around $24 \mathrm{kHz}$ ) can be transmitted simultaneously with a high frequency pulse (normally around $200 \mathrm{kHz}$ ). Because these frequencies are discrete, there is not interference between the two return signals. Dual frequency echo sounding produces positive effects, such as the facility to recognize a sea grass (Posidonia Oceanica) layer. The high frequencies are appropriate in shallow water [23]. The lower frequencies are suitable in deeper water because less susceptible to attenuation in the water column [24].

Most hydrographic operations use a $200 \mathrm{kHz}$ transducer, which is suitable for inshore work up to 100 meters in depth. Deeper water requires a lower frequency transducer as the acoustic signal of lower frequencies is less susceptible to attenuation in the water column. Commonly used frequencies for deep water sounding are $33 \mathrm{kHz}$ and $24 \mathrm{kHz}$.

The beam width of the transducer determines the resolution of the data, so a fine one is preferable. This aspect is fundamental for hydrographic survey in deep water, because the resultant footprint of the acoustic pulse can become too much great once it reaches a far object.

Single beam echo sounder must be calibrated by a bar check for correct determination of the speed of sound in the water column [25]. The bar check consists of immersing a flat plate below the echo sounder transducer, measuring the depths at points of known depths below the surface and comparing the actual and measured depths. The sound velocity in the echo sounder is modified until the measured depth is equal the known depth [26]. Finally, the echo sounder is fixed with the average sound velocity over the water column.

A bar check should be conducted at least daily, and whenever there is a change of survey area during the day, to ensure consistent data quality [27]. Likewise, the use of bar check is necessary when any SBES components are modified or replaced on the vessel.

\section{B. Study Area and Pre-Elaboration of Depth Data}

For this study, 2023 depth points resulting from single beam echo sounder survey of the Gulf of Pozzuoli, are used for 3D bathymetric models construction. Depth measurement data are kindly provided by the Italian Hydrographic Office (Istituto Idrografico della Marina) and present decimeter accuracy. The interested area is reported in Fig. 1. It extends within the following UTM/WGS84 plane coordinates - 33T zone: $\mathrm{E}_{1}=$ $423,630 \mathrm{~m}, \mathrm{E}_{2}=428,320 \mathrm{~m}, \mathrm{~N}_{1}=4,513,461 \mathrm{~m}, \mathrm{~N}_{2}=4,518,761$ $\mathrm{m}$. Depth values range between $-9.7 \mathrm{~m}$ and $-118.1 \mathrm{~m}$.

Because the sample points are fairly evenly distributed along the ship routes and these are not very far each from others, the distribution of depth values is quite uniform, as it is shown in Fig. 2.

Depth data are available in txt format and converted in vector file (shp) using the ESRI software ArcGIS 10.3 [28]. The extension named "Geostatistical analyst" and available within the above mentioned program, is used to generate bathymetric models of the study area.

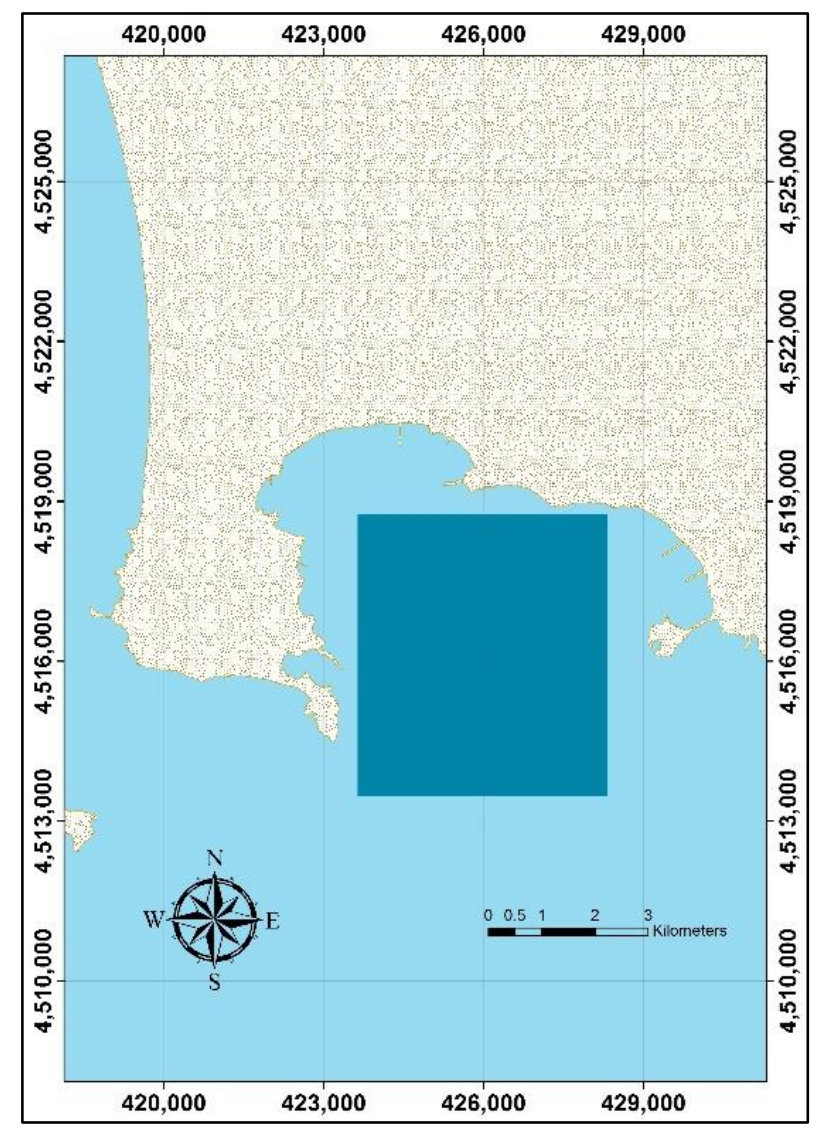

Fig. 1. The Gulf of Pozzuoli: in Blue the Area Covered by Single Beam Echo Sounder Data. 


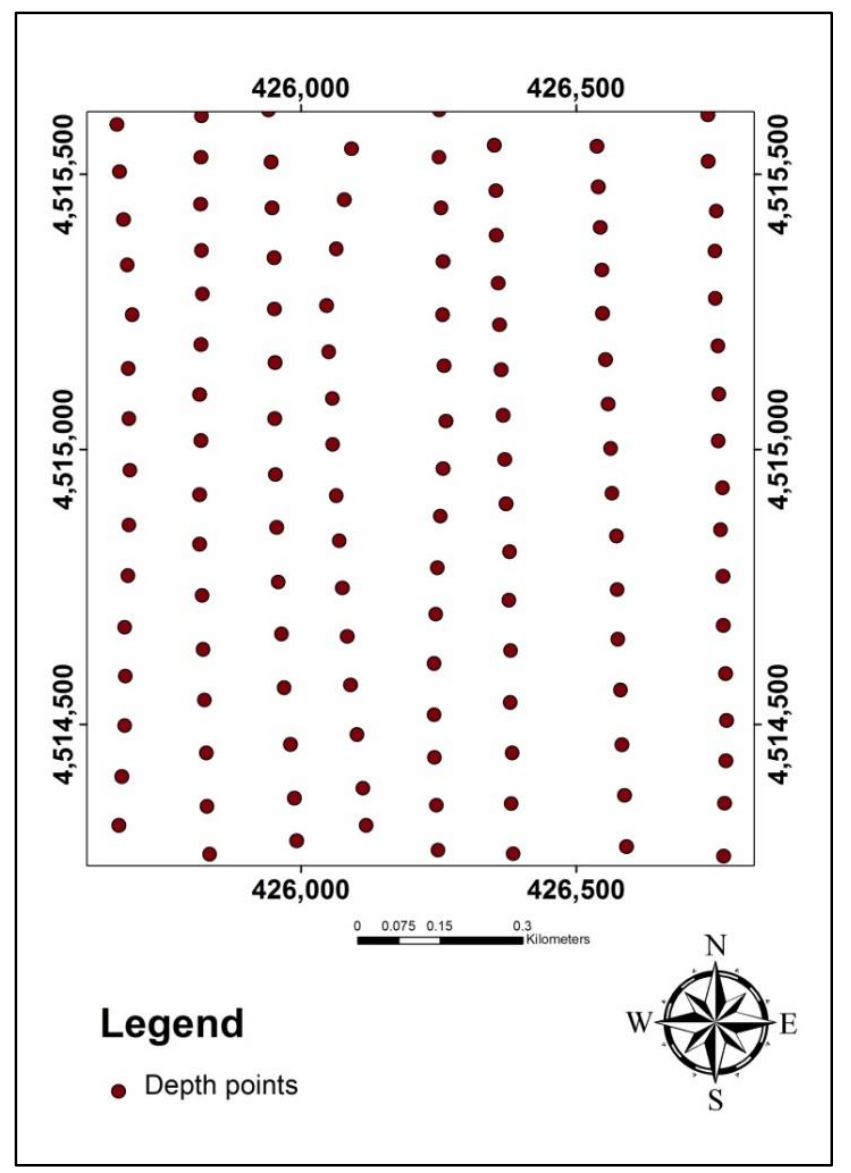

Fig. 2. Particular of the Distribution of the Depth Points.

\section{INTERPOLATION METHODS}

In literature different interpolation methods are available and their application to the same data can produce different results. Therefore, it is necessary to compare these methods and choice the most performed one.

The interpolation methods are founded on the principle of spatial autocorrelation, which is well represented by Tobler's first law of geography: "Everything is related to everything else, but near things are more related than distant things" [29, 30].

Methods of interpolation are generally classified into two categories: local and global methods. In the local methods, the interpolated value is affected only by the values at nearby points from the initial data set, while in the global methods, each interpolated value is affected by all of the data [31]. For local methods, a specified number of points, or all points within a specified radius, are used to determine the output value of each location. In Fig. 3, the research of the neighbouring measured points using a specified radius is shown.

Another way to classify interpolation methods is to distinguish them as either exact or approximate methods, according to their characteristic of preserving or not preserving the original sample point values on the inferred surface [32].

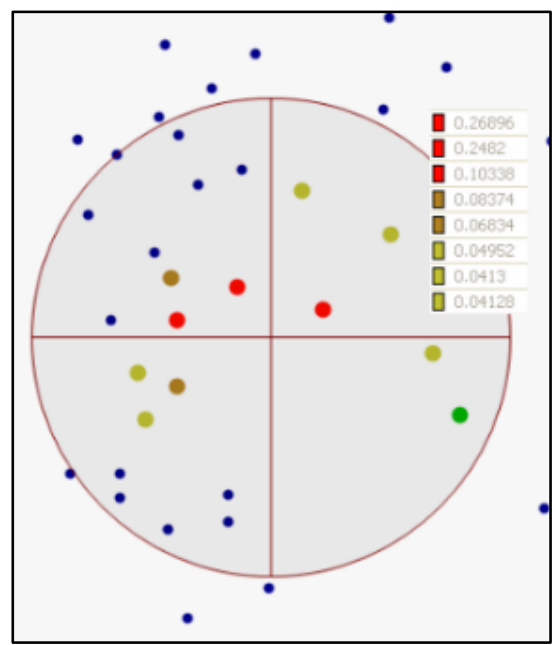

Fig. 3. The Research of the Neighbouring Measured Points (Source: ArcGIS Pro Help).

This section briefly presents the different interpolation methods used in this study. For all local interpolators, to determine the output value for each location, four sectors are used, with maximum number of points equal to 15 neighbours, and minimum equal to 10 for each sector.

\section{A. The IDW method}

Inverse Distance Weighting (IDW) is a deterministic interpolation method, so the resulting surface must pass through each measured sample value. This method is based on the assumption that closer values are more related than further values. It uses measured values surrounding an unmeasured location to predict its value [32].

Interpolated points are estimated based on their distance from known values: for consequence, points that are adjacent to known values are more influenced than points that are distant [33]. In other terms, the values of the neighbouring measured points are weighted by the inverse of the distance at the calculated [34]. As a result, as the distance increases, the weights decrease rapidly [35].

The interpolation function is represented by the following formula:

$z_{x, y}=\frac{\sum_{k=1}^{N} \frac{z_{k}}{d_{k}^{p}}}{\frac{1}{d_{k}^{p}}}$

Where

$\mathrm{Z}_{\mathrm{x}, \mathrm{y}}=$ estimated value at the position $(\mathrm{x}, \mathrm{y})$ of the grid,

$\mathrm{Z}_{\mathrm{k}}=$ a neighbouring data point value,

$\mathrm{N}=$ the number of neighbouring points,

$\mathrm{d}_{\mathrm{k}}=$ the distance between the data point and the point being interpolated,

$\mathrm{P}=$ a positive-power parameter.

In this study, IDW with power equal to 2 is applied. 


\section{B. Radial Basis Function (RBF)}

Radial basis functions (RBFs) are a series of exact interpolation techniques, so the surface passes through the data values.

There are different basis functions, such as: completely regularized spline (RBF-CRS), spline with tension (RBFSwT), thin-plate spline (RBF-TPS), multiquadric function (RBF-MF), inverse multiquadric function (RBF-IMF). Each basis function produces a different interpolation surface [36].

A RBF is conceptually similar to an elastic membrane that fits on the measured sample values and minimizes the total curvature of the surface; the selection of the basis function determines how the rubber membrane fits between the values $[37,38]$.

In this study, all the above five mentioned RBFs are applied.

\section{Global Polynomial Interpolation}

Global polynomial interpolation (GPI) is an approximate method that fits a smooth surface defined by a mathematical function (a polynomial) to the input sample points [39].

The user can choice the order of the polynomial that ranges from a first-order to higher order. The interpolation function can be written as:

$z=\sum_{i=0}^{m_{1}} \sum_{j=0}^{m_{2}} a_{i, j} x^{i} y^{j}$ valid:

If $\mathrm{n}$ is the order of the equation, the following relations are

$0 \leq m_{1} \leq n$

$0 \leq m_{2} \leq n$

$m_{1}+m_{2} \leq n$

The values of the coefficients $a_{i, j}$ are determined using the known elevation values in the sample points.

The predictive surface is typically generated by using a least-square regression fit that minimizes the squared differences between the surface and measured points [40]. The estimation of the coefficients permits to determine the value of the polynomial function at any point within the map area [41].

The polynomial can be expanded to any desired degree, although there are computational limits because of rounding error. In this study, the orders 1, 2 and 3 are considered. As we all know, a first order polynomial (linear) corresponds to a flat plan (no bend). A second-order polynomial (quadratic) allows for one bend, a third-order (cubic) for two bends [39].

\section{Local Polynomial Interpolation}

Local polynomial interpolation (LPI) is similar to GPI, except that it uses a local subset defined by a window rather than using the entire dataset [42]. The window is shifted across the map area and the surface value at the centre of the window is estimated. The size of this window must be large enough to include a reasonable number of data point [43]. For example, a second order polynomial requires at least six points, a third order ten points, and so on.
LPI is more flexible than GPI, but requires to define more parameters [42], i.e. the neighborhood shape, maximum and minimum number of points, sector configuration [44].

\section{E. Kriging}

Kriging originated in the field of mining geology as is named after Danie Gerhardus Krige, a mining engineer born in Bothaville, Free State, South Africa, in 1919 [45].

Similarly to IDW, Kriging weights the surrounding measured values to estimate the value at an unknown point. Unlike IDW and other methods, Kriging uses the spatial correlation between sampled points to estimate the value at an unknown point: the spatial arrangement among the measured points, rather than a presumed model of spatial distribution, is used for interpolation; in addition to that, it supplies estimates of the uncertainty surrounding each interpolated value [46].

In other terms, in Kriging, the weights are founded not only on the distance between the measured points and the estimate location, but also on the overall spatial organization of the empirical observations; this can be introduced using the spatial autocorrelation [47].

The spatial correlation between the measurement points can be computed by means of the semi-variance function. It is half the variance of the differences between all possible points spaced a constant distance apart:

$\gamma=\frac{1}{2 N(h)} \sum_{i=1}^{N(h)}\left[Z\left(u_{i}\right)-Z\left(u_{i}+h\right)\right]^{2}$

Where

$\mathrm{N}(\mathrm{h})$ is the number of pairs of measurement points with distance $\mathrm{h}$ apart;

$z\left(u_{i}\right)$ is the value at location $u_{i}$,

$z\left(u_{i}+h\right)$ is the value at location $u_{i}+h$

As points are compared to increasingly distant points, the semivariance increases; in case of strong spatial dependence, points that are closer together will present a smaller semivariance [48].

A variogram is introduced as a graphical representation of the covariance calculated between each pair of points in the sampled data and plotted against the distance. Because half the variance is plotted, the resulting graphical representation is sometimes called "semivariogram".

Usually, to speed up the procedure, the pairs are gathered into lag bins. For example, the average semivariance is calculated for all pairs of points that present distance between 20 meters and 30 meters.

The empirical semivariogram plotting the observed values is substituted with an acceptable semivariogram model that best fits the data. In this way, the kriging algorithm can access to semivariogram values for lag distances other than those used in the empirical semivariogram [49].

There are several Variogram models available to fit the data [50,51], i.e. linear, gaussian, exponential, stable, etc. 


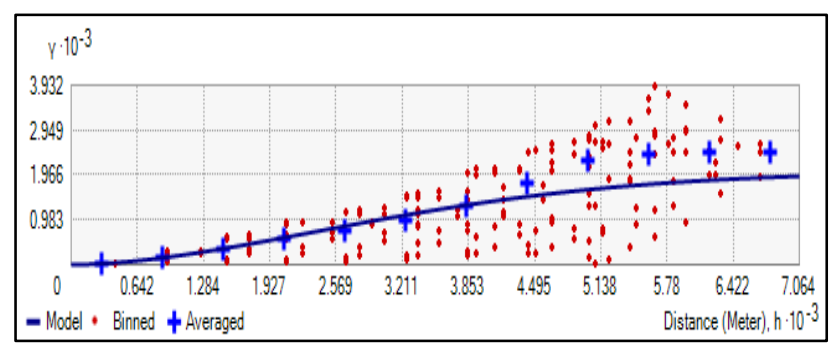

Fig. 4. The Semi-Variogrm Obtained with Ordinary Kriging Application to Bathymetric Data.

Different types of kriging are present in literature, and interested readers could refer to several papers for detailed description of them, e.g. [52, 53]. In this study ordinary kriging (OK), simple kriging (SK) and universal kriging (UK) are considered, using in all cases stable variogram model because it produces the best results. The resulting semi-variogram obtained with ordinary kriging, is shown in Fig. 4.

\section{COMPARISON OF METHODS}

The accuracy of an interpolation method is usually tested analyzing the closeness of interpolated values to accepted values. In the case of DDM, measured depths can be considered the accepted values. However, the analysis should be carried out on Check Points (CPs) that are not listed in the dataset used for interpolation, and thus the amount of information to be used for DDM generation would be depleted. In other terms, the lack of knowledge about the depth in some points used as CPs would limit the accuracy of the resulting model in these points.

For this reason, the best approach to evaluate the accuracy of DDM is to use Cross Validation (CV). Different methods are available in literature for CV application [54].

One of the possible approach for $\mathrm{CV}$ is the leave-one-out method: each sampling point is removed from the dataset and the other points are used to interpolate the depth value at its location; the residual is calculated between measured and interpolated values before moving to the next point [55,56,57]. Rather than removing all the points, once at time, a subset of the initial database can be removed in turn [58]. For this study, the leave-one-out method is applied. The overall performance of the interpolation methods can be avaluated using statistical terms of the differences between the original and interpolated points, such as mean, minimum, maximum, mean absolute, Root Mean Square Error (RMSE), and other factors [59]. RMSE is expressed as:

$R M S E=\sqrt{\frac{\sum_{i=1}^{N}\left(Z_{i}(x, y)-Z_{i}^{\prime}(x, y)\right)^{2}}{N}}$

Where:

$\mathrm{N}$ is the number of the depth points;

$\mathrm{Zi}(\mathrm{x}, \mathrm{y})$ is the measured depth at the location $\mathrm{i}(\mathrm{x}, \mathrm{y})$;

$\mathrm{Z}_{\mathrm{i}}^{\prime}(\mathrm{x}, \mathrm{y})$ is the interpolated depth at the same location $\mathrm{i}(\mathrm{x}, \mathrm{y})$.

In this study, fifteen interpolation methods are applied. In Table I, minum, maximum, mean and RMSE of the residuals calculated by $\mathrm{CV}$ for each method is reported.
By comparing the interpolation methods applied in this study, it is possible to note some significant differences as well as analogies in the output results for each statistic parameter. The range of minimum values (in $\mathrm{m}$ ) goes from -44.304 (GPI1) to -8.991 (SK), while the range of maximum values (in m) goes from 5.054 (RBF) to 22.352 (GPI-1). The range of mean values (in $\mathrm{m}$ ) goes from -0.038 (IDW) to 0.536 (SK), while the range of RMSE goes from 13.682 to 0.608 (RBF-SwT and RBF-TPS). According to all indicators, four methods tend to be equal in terms of better performance: RBF-SwT, RBF-TPS, OK and UK.

Generally, RBF interpolation methods tend to produce good results for gently varying surface: they are inappropriate when large changes in the depth values occur within short distances and/or when the sample data are affected by considerable measurement error or uncertainty [36]. The area considered in this study is free of wide variations in the morphology and the depth measurements are sufficiently accurate, so RBF methods produce excellent results.

The appropriateness of Kriging approach for bathymetry representation and its better performance that other methods such as IDW [60] are confirmed in this study.

As is to be expected, GPIs give the worst outcomes. As evidenced by other performing methods, the study area presents a morphology that is unlike plane or second or third order surface, so the results of GPIs cannot be satisfactory.

One of the most performed model, resulting from ordinary kriging application, is shown in Fig. 5 (2D visualization) and Fig. 6 (3D visualization).

TABLE. I. Statistical Terms of THE Residuals SupPlied by CRoss VALIDATION

\begin{tabular}{|l|l|l|l|l|}
\hline \multirow{2}{*}{$\begin{array}{l}\text { Interpolation } \\
\text { method }\end{array}$} & \multicolumn{4}{|l|}{ Statistical terms of the residuals } \\
\cline { 2 - 5 } & Min $(\boldsymbol{m})$ & Max $(\boldsymbol{m})$ & Mean $(\boldsymbol{m})$ & RMSE $(\boldsymbol{m})$ \\
\hline IDW & -12.982 & 6.251 & -0.038 & 1.517 \\
\hline RBF - CRS & -14.707 & 9.538 & -0.010 & 1.201 \\
\hline RBF - SwT & -9.313 & 5.054 & 0.004 & 0.608 \\
\hline RBF - TPS & -9.313 & 5.054 & -0.003 & 0.608 \\
\hline RBF - MF & -9.381 & 7.372 & -0.009 & 0.801 \\
\hline RBF - IMF & -9.449 & 7.934 & -0.007 & 0.876 \\
\hline GPI-1 & -44.304 & 22.352 & 0.006 & 13.682 \\
\hline GPI-2 & -35.367 & 17.129 & 0.002 & 5.840 \\
\hline GPI-3 & -30.502 & 12.297 & -0.001 & 4.350 \\
\hline LPI-1 & -9.347 & 5.198 & 0.058 & 0.735 \\
\hline LPI-2 & -9.272 & 5.809 & 0.006 & 0.757 \\
\hline LPI-3 & -9.197 & 5.667 & -0.006 & 0.748 \\
\hline OK & -9.335 & 5.272 & 0.027 & 0.724 \\
\hline SK & -8.991 & 8.006 & 0.536 & 1.649 \\
\hline UK & -9.332 & 5.272 & 0 & 0.717 \\
\hline
\end{tabular}




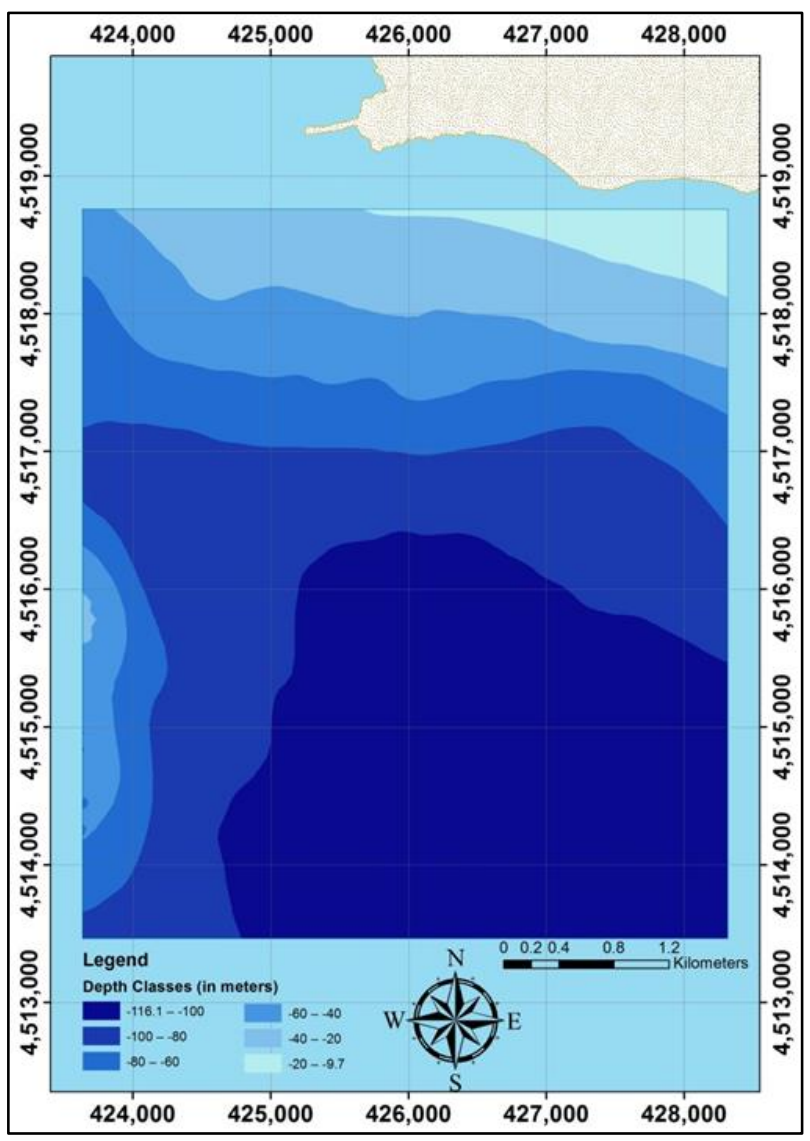

Fig. 5. One of the Most Performed Model (Resulting from Ordinary Kriging Application) in 2d Visualization.

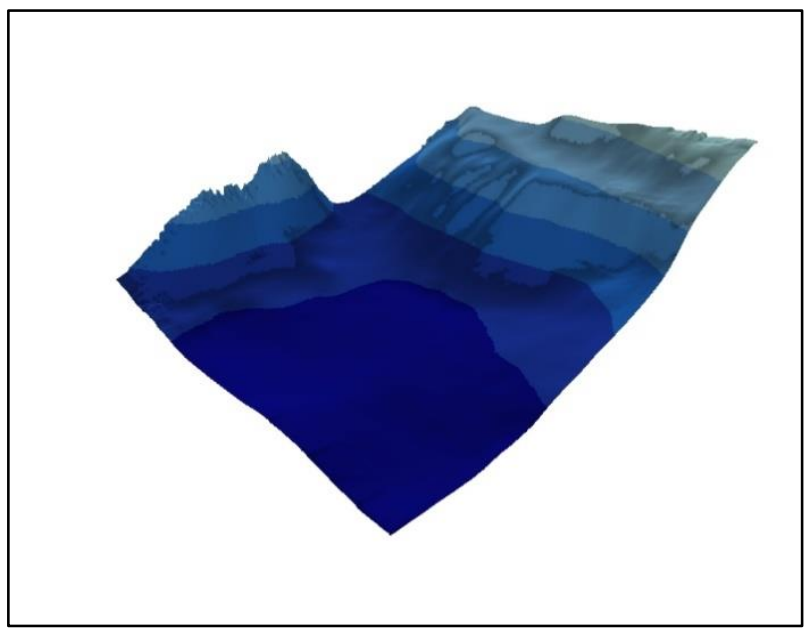

Fig. 6. 3D Visualization of the Model Shown in Fig. 5.

\section{CONCLUSION}

The analysis presented here illustrates the performance of spatial interpolation methods for bathymetry modelling. Because every method gives a different output representation, the main challenge is to produce the most accurate model based on the available data. This goal can be achieved by comparing different methods. In this study, 15 methods are tested on 2023 depth points resulting from single beam echo sounder survey of the Gulf of Pozzuoli. CV leave-one-out is used to analyze the performance of each interpolation methods: statistical terms of the differences between measured and interpolated points permit to compare the considered methods.

RBF-SwT, RBF-TPS, OK and UK present the better performance, GPI-1, GPI-2 and GPI-3 the worst. The positive results of some interpolation methods find easy explanations: they are due to the quite regular distribution and sufficiently accurate measurement of the initial points for the $\mathrm{RBF}$ methods, and to the greater interpretative capacity of the stochastic approach for kriging methods.

The results obtained in this study remark that the quality of a DDM is related to the choice of an appropriate interpolation method in order to fit the dataset, according to other studies on those aspects $[11,34,42]$. However, single beam echo sounder survey permits the availability of data that are not completely random: depth points are quite regularly distributed along the ship routes and that eases the interpolation process. Nevertheless, some factors such as the distance between two nearby ship routes and seabed morphology have an important influence on the accuracy of the chosen method.

Concerning the future developments of this work, further studies will be focused on the possibility to integrate data from different bathymetry survey in order to increase the number of available depth values and to evaluate the relationship between point density and model accuracy. In addition, we will be mainly focused on the relationship between interpolation methods and seabed morphology.

\section{ACKNOWLEDGMENT}

The research is supported by University of Naples "Parthenope". We wish to thank the Italian Hydrographic Office (Istituto Idrografico della Marina) for providing the data. We also like to thank the technical staff of our Department for their contribution to this project; particularly, special thanks should be given to Mr. Mariano Rovito (Laboratory of Topography and Photogrammetry) and Ferdinando Sposito (Laboratory of Geomatica, Remote sensing and GIS), for their valuable technical support to this project.

\section{REFERENCES}

[1] Z. Zhan, Y. Fu, R. J. Yang, Z. Xi, Z., and L. Shi, "A Bayesian inference based model interpolation and extrapolation," SAE International journal of materials and manufacturing, 5(2), pp. 357-364, 2012.

[2] K. Wang, "A study of cubic spline interpolation," InSight: Rivier Academic Journal, 9(2), 2013.

[3] P. P. Shingare, and M, S. S, Kale, "Review on digital elevation model," International Journal of Modern Engineering Research (IJMER), 3(4), pp. 2412-2418, 2013.

[4] A. Barac, Automatic conversion of scanned sea charts into 3D models (Master's thesis), 2012.

[5] J. Stoer, and R. Bulirsch, Introduction to numerical analysis, Vol. 12, Springer Science \& Business Media, 2013.

[6] S. D. Conte, and C. De Boor, Elementary numerical analysis: an algorithmic approach, SIAM, Philadelphia (USA), 2017.

[7] C. L. Miller, and R. A. Laflamme, "The digital terrain model theory and application," Photogrammetric Engineering, Vol. 24 (Issue 3), pp- 433442, 1958.

[8] Stilla, U., and Soergel, U. "Reconstruction of buildings in SAR imagery of urban areas," in Urban remote sensing (pp. 47-67). Taylor \& Francis, 2014. 
[9] C. Hirt, "Digital terrain models," in Encyclopedia of Geodesy, 1-6, DOI 10.1007/978-3-319-02370-0_31-1, Springer International Publishing, Switzerland, 2014.

[10] Z. Li, Q. Zhu, and C. Gold, Digital terrain modeling: principles and methodology, CRC Press, Boca Raton, FL, 2005.

[11] G. Garnero, and D.Godone, "Comparisons between different interpolation techniques," in International Archives of the Photogrammetry, Remote Sensing and Spatial Information Sciences, 5, W3, 2013.

[12] P. Maglione, C. Parente, R. Santamaria, and A. Vallario, "3D thematic models of land cover from DTM and high-resolution remote sensing images WorldView-2," Rendiconti Online della Società Geologica Italiana, Vol. 30:33-40, 2014.

[13] N. A. Gesim, N. A., and T. Okazaki, "Assessment of Groundwater Vulnerability to Pollution using DRASTIC Model and Fuzzy Logic in Herat City, Afghanistan," IJACSA - International Journal of Advanced Computer Science and Applications, 9(10), pp. 181-188, 2018.

[14] A. Rasooli, and D. Kang, "Assessment of potential dam sites in the Kabul River Basin using GIS," IJACSA - International Journal of Advanced Computer Science and Applications, Vol. 6, No. 2, 2015.

[15] M. C. Spreafico, L. Perotti, F. Cervi, M. Bacenetti, G. Bitelli, V. A. Girelli, E. Mandanici, M. A. Tini, and L. Borgatti, "Terrestrial Remote Sensing techniques to complement conventional geomechanical surveys for the assessment of landslide hazard: The San Leo case study (Italy)," European Journal of Remote Sensing, 48(1), pp. 639-66., 2015.

[16] K. Freeborough, C. Dashwood, D. D. Doce, G. Jessamy, S. Brooks, H. Reeves, and S. Abbott, "A national assessment of landslide hazard from Outside Party Slopes to the rail network of Great Britain," Quarterly Journal of Engineering Geology and Hydrogeology, qjegh2018-029, 2018.

[17] P. Maglione, C. Parente, and A. Vallario, "High resolution satellite images to reconstruct recent evolution of domitian coastline," American Journal of Applied Sciences, 12(7), pp. 506-515, 2015.

[18] International Hydrographic Organization (IHO), IHO Standards for Hydrographic Surveys. 5th edition, International Hydrographic Bureau, Monaco, Special Publication No: 44, 2008.

[19] C.T. Chen, and F. J Millero, "Speed of sound in seawater at high pressures," The Journal of the Acoustical Society of America, vol. 62, no. 5, pp. 1129-1135, 1977.

[20] R. Urick, Principles of Underwater Sound, McGraw-Hill, 1983.

[21] F. J. L. Ribeiro, A. C. P. Pedroza, and L. H. M. K. Costa, "Deepwater monitoring system using logistic-support vessels in underwater sensor networks," in The 21st International Offshore (Ocean) and Polar Engineering Conference-ISOPE, Vol. 2, pp. 327-333, 2011.

[22] Nichy Du, "Speed of Sound in Water," in The Physics Factbook Hypertextbook,2000,https://hypertextbook.com/facts/2000/NickyDu.sht $\mathrm{ml}$.

[23] F. Giordano, G. Mattei, C. Parente, C., F. Peluso, and R. Santamaria, "Integrating sensors into a marine drone for bathymetric 3D surveys in shallow waters". Sensors, 16(1), 41, 2016.

[24] P Barr, F. J., and Sanders, J. I. "Attenuation of water-column reverberations using pressure and velocity detectors in a water-bottom cable," in SEG Technical Program Expanded Abstracts 1989 (pp. 653656). Society of Exploration Geophysicists, 1989.

[25] The International Federation of Surveyors (FIG), Publication 56 Guidelines for the Planning, Execution and Management of Hydrographic Surveys in Ports and Harbours, Copenhagen, Denmark, 2010

[26] Ferreira, H., Almeida, C., Martins, A., Almeida, J., Dias, N., Dias, A., and Silva, E. "Autonomous bathymetry for risk assessment with ROAZ robotic surface vehicle," in Oceans 2009-Europe (pp. 1-6). Ieee.

[27] Canadian Hydrographic Service, Hydrographic survey management guidelines, June 2013, Edition 2, 2009.

[28] Esri, ArcGIS 10.3, Redlands, CA, USA, 2015.

[29] W. R. Tobler, "A computer movie simulating urban growth in the Detroit region," Economic Geography, Vol. 46, pp. 234-40, 1970.

[30] H. J. Miller, "Tobler's first law and spatial analysis," Annals of the Association of American Geographers, 94(2), pp. 284-289, 2004.
[31] C. Özkan, "Surface interpolation by adaptive neuro-fuzzy inference system based local ordinary kriging," in Asian Conference on Computer Vision, pp. 196-205, Springer, Berlin, Heidelberg, January 2006,

[32] N. S. N. Lam, "Spatial interpolation methods: a review," The American Cartographer, 10(2), pp. 129-150, 1983.

[33] Bartier, P. M., and Keller, C. P.. Multivariate interpolation to incorporate thematic surface data using inverse distance weighting (IDW). Computers \& Geosciences, 22(7), 795-799, 1996.

[34] Alcaras, E., Parente, C. and Vallario, "A Comparison of different interpolation methods for DEM production," International Journal of Advanced Trends in Computer Science and Engineering. Vol. 6: 16541659, 2019.

[35] ESRI, "How inverse distance weighted interpolation works," in ArcGIS Pro help, ESRI, Redlands, CA, USA.

[36] ESRI, "How radial basis functions work," in ArcGIS Pro help, ESRI, Redlands, CA, USA.

[37] G.F. Lin, and L.H. Chen, "A spatial interpolation method based on radial basis function networks incorporating a semivariogram model,". J. Hydrol 288(3-4), pp.288-298, 2004, doi:10.1016/j.jhydrol.2003.10.008

[38] H. Apaydin, F. K. Sonmez, and Y. E. Yildirim, "Spatial interpolation techniques for climate data in the GAP region in Turkey," Climate Research, 28(1), pp. 31-40, 2004.

[39] ESRI, "How global polynomial interpolation works," in ArcGIS Pro help, ESRI, Redlands, CA, USA,

[40] S. Eberly, J. Swall, D. Holland, B. Cox, and E.Baldridge, ,'Developing spatially interpolated surfaces and estimating uncertainty," United States Environmental Protection Agency, pp. 28-40, 2004.

[41] W. Wang, W. Qi, N. Chen, P. Wang, and J. Chen, J. "Research on determining the weights of urban land grading evaluation factors based on Spearman rank correlation analysis," in International Symposium on Spatial Analysis, Spatial-Temporal Data Modeling, and Data Mining, Vol. 7492, p. 74921U, International Society for Optics and Photonics, October 2009.

[42] W. Luo, M. C. Taylor, and S. R. Parker, "A comparison of spatial interpolation methods to estimate continuous wind speed surfaces using irregularly distributed data from England and Wales," International Journal of Climatology: A Journal of the Royal Meteorological Society, 28(7), 947-959, 2008.

[43] M. J. De Smith, M. F. Goodchild, and P. Longley, Geospatial analysis: a comprehensive guide to principles, techniques and software tools. Troubador publishing ltd, Leichester (UK), 2007.

[44] J. Seyedmohammadi, L. Esmaeelnejad, M. Shabanpour, "Spatial variation modelling of groundwater electrical conductivity using geostatistics and GIS," Modeling earth systems and environment, 2(4), pp. 1-10, 2016.

[45] Assibey-Bonsu, W., "Professor Danie Krige's First Memorial Lecture: The Basic Tenets of Evaluating the Mineral Resource Assets of Mining Companies, as Observed in Professor Danie Krige's Pioneering Work Over Half a Century," in Geostatistics Valencia 2016 (pp. 3-25). Springer, Cham, 2017.

[46] Columbia University Mailman School of Public Health, Kriging, Columbia University, 722 West 168th St. NY, NY 10032, https://www.mailman.columbia.edu/research/population-health-methods /kriging

[47] ESRI, "How kriging works", in ArcGIS Pro help, ESRI, Redlands, CA, USA,

[48] L. H. Peters, Spatial Autocorrelation: Covariance and Semivariance, GEOG 593, 2009.

[49] G. Bohling, G., "Introduction to geostatistics and variogram analysis," Kansas geological survey, 1, pp. 1-20.

[50] M. Armstrong, Basic linear geostatistics. Springer Science \& Business Media, 1998.

[51] D. Chen, Advances in data, methods, models and their applications in Geoscience. BoD-Books on Demand, 2011.

[52] M Meul, and M. Van Meirvenne, "Kriging soil texture under different types of nonstationarity," Geoderma, 112(3-4), pp. 217-233, 2003. 
[53] P. Singh, and P. Verna, "A Comparative Study of Spatial Interpolation Technique (IDW and Kriging) for Determining Groundwater Quality," in Venkatramanan, S., Viswanathan, P. M., \& Chung, S. Y. (Eds.). GIS and Geostatistical Techniques for Groundwater Science, Elsevier, 2019.

[54] K. C. Lam, R. G. Bryant, and J. Wainright, "Application of spatial interpolation method for estimating the spatial variability of rainfall in Semiarid New Mexico, USA," Mediterranean Journal of Social Sciences, 6(4), S3, 2015.

[55] P. A. Burrough, and R.A. McDonnell, Principles of Geographical Information Systems, Oxford: Oxford University Press, 1998.

[56] R. S. Bivand, E.J. Pebesma, and V. Gomez-Rubio, Applied Spatial Data Analysis with R, Springer, 2008.
[57] G. E.Fasshauer, and J. G. Zhang, "On choosing "optimal" shape parameters for RBF approximation," Numerical Algorithms, 45(1-4),pp. 345-368, 2007.

[58] U. Falchi, C. Parente, and G. Prezioso, "Global geoid adjustment on local area for GIS applications using GNSS permanent station coordinates," Geodesy and Cartography, 44(3), pp. 80-88, 2018.

[59] A. I. EL-Hattab, "Single beam bathymetric data modelling techniques for accurate maintenance dredging," The Egyptian Journal of Remote Sensing and Space Science, 17(2), 189-195, 2014.

[60] I. O. Ferreira, D. D. Rodrigues, G. R. D. Santos, and L. M. F. Rosa, "In bathymetric surfaces: idw or kriging?," Boletim de Ciências Geodésicas, 23(3), pp. 493-508, 2017. 